\title{
The changing patterns of access overtime to the renal replacement therapy programme in Thailand
}

\author{
Authors \\ Noppakun Thammatacharee ${ }^{1 /} \quad$ PhD noppakun@hsri.or.th \\ Anne Mills $^{2 /} \quad$ PhD Anne.Mills@1shtm.ac.uk \\ Dorothea Nitsch $^{2 /} \quad$ MSc Dorothea.Nitsch@1shtm.ac.uk \\ Adisorn Lumpaopong ${ }^{3 /} \quad$ MD adisornuic@yahoo.com \\ ${ }^{1 /}$ Health Systems Research Institute and International Health Policy Program, Thailand \\ ${ }^{2 /}$ London School of Hygiene and Tropical Medicine, UK \\ ${ }^{3 /}$ Phramongkutkao Hospital, Thailand
}

Corresponding author: Noppakun Thammatacharee, Health Systems Research Institute, 88 Satharanasuk 6 Alley, Mueang, Nonthaburi 11000

Telephone: +66(0)85118 4543, Fax: +66(0)2 8329201

Keywords: renal replacement therapy, dialysis, kidney transplant, universal health coverage, age-period-cohort analysis

\section{Key Messages}

- Arrangements of an RRT programme resulted in an increased access to high-cost care in Thailand.

- Dialysis registration was increasing in young patients.

- Dialysis and kidney transplantation in elderly were low and tended to decrease with age.

\begin{abstract}
Based on projected numbers, approximately only $50 \%$ of those requiring renal replacement therapy (RRT) receive it. Many patients who require RRT live in low and middle income countries. The objective of this study was to examine the changing pattern over time of entry
\end{abstract}


into the renal replacement therapy programme in Thailand following RRT's inclusion in the Universal Coverage Scheme. This study was an ecological study using the age-period-cohort analysis to look at dialysis registration and kidney transplant trends during RRT programme implementation. Data from 2008-2016 of patients diagnosed with end-stage renal disease (ESRD) were obtained from the National Health Security Office. The study found that the numbers of new patients with ESRD, aged 20-69, registered with the dialysis programme increased over time. For patients aged 20-40 years, the dialysis programme took up to 400 new patients for every 1,000 new ESRD diagnoses. For kidney transplant, the rates increased slowly. The kidney transplant programme could at best treat only around 50 cases for every 1,000 new ESRD diagnoses in patients aged 20-30 years. Findings of this study highlighted the importance of promoting strategies to reduce the increasing number of patients with kidney disease, to consider conservative therapy for older/frail patients, and improve access to kidney transplantation and live-donation. 


\section{Introduction}

Chronic kidney disease has been increasingly recognised as a global public health problem, not only because of the rising prevalence across the world but also because of widening inequities in accessing renal replacement therapy (RRT) when kidney function has failed completely (Grassmann et al. 2005; White et al. 2008; Coresh and Jafar 2015; Liyanage et al. 2015). It is estimated that, worldwide, only a half of those needing RRT receive it. Of those receiving RRT, only 9\% reside in low- and middle-income countries, yet these people make up at least $38 \%$ of those who need the treatment (Liyanage et al. 2015). In these countries, the low percentage of people accessing treatment is a consequence of the unaffordable cost of dialysis (Jah 2013), arrangements of RRT service provision (Odubanjo, Oluwasola et al. 2011), and variation in universal public reimbursement for services (Sakhuja and Kohli 2006; Pecoits-Filho et al. 2009).

The Thai government launched the Universal Coverage Scheme (UCS) in 2001 to cover previously uninsured citizens outside the other two public schemes, namely, the Civil Servant Medical Benefit Scheme and the Social Security Scheme. To date, the UCS covers 48 million beneficiaries or $76 \%$ of Thais (NHSO 2017). RRT was initially excluded from the UCS benefit package due to fiscal constraints and lack of treatment facilities. The National Health Security Office (NHSO), which was responsible for the UCS, was increasingly pressured to expand benefits to include RRT, both dialysis and transplantation, by civil society organisations and patient groups (Tangcharoensathien et al. 2005). Despite being known to have poor cost-effectiveness relative to many other interventions (Sennfalt et al. 2002; Kontodimopoulos and Niakas 2008; Haller et al. 2011), in 2008 RRT was adopted into the benefit package of the UCS on the grounds that it would save lives and prevent indebtedness and health impoverishment among UCS members. The UCS introduced the RRT programme 
as a so-called 'disease management programme' which would provide RRT services for every UCS member in need.

The UCS-funded RRT adopted a 'PD first' policy, meaning that all new end-stage renal disease (ESRD) patients without contraindications must use continuous ambulatory peritoneal dialysis (CAPD) as first-line therapy, or shoulder the costs of hemodialysis (HD) themselves. HD patients who were on HD before the 'PD first' policy, and patients with contraindications to CAPD, were eligible for full reimbursement of the cost of HD. Under the PD-first policy, renal transplant and all essential high-cost medications are also included in the benefits (Chungsaman and Kasemsap 2017). When patients' eGFR level reaches 15 $\mathrm{ml} /$ minute per $1.73 \mathrm{~m}^{2}$, they are diagnosed as ESRD. However according to the Thai Nephrology Society guidelines, UCS patients without signs or symptoms of kidney failure will be asked to enter the RRT programme once the eGFR level drops to $6 \mathrm{ml} / \mathrm{minute}$ per $1.73 \mathrm{~m}^{2}$

Thailand, as a developing country, faces problems of inadequacy of health care infrastructure. To date, there has been limited study of the performance of the RRT programme. This study aimed to assess the changing patterns of entry into the RRT programme of adult UCS patients diagnosed with ESRD using an age-period-cohort analysis to establish whether launching the RRT programme promoted entry into the programme over time.

\section{Methods}

\section{Data sources}

We undertook the age-period-cohort analysis using the administrative health databases of the UCS. These databases contain detailed demographic, diagnostic, procedures, medications, laboratory, and other clinical data at the individual level, and can be linked together and also 
linked to mortality data from the Ministry of Interior's civil registration system by using the 13-digit citizen identification number. The NHSO has audit procedures to ensure quality as well as prevent duplicates of the claims data at both central and regional NHSO, and also has encoding processes for all citizen identification numbers before handing the data to any third party.

\section{Study population}

A cohort of patients who were diagnosed with ESRD was first constructed. It included adult UCS members aged 20 to 69 who had an index hospitalisation or outpatient visit with an ESRD diagnosis (ICD-10 code N180 or N185) as either primary diagnosis or secondary diagnosis between 1 January 2008 (the date RRT was adopted into the UCS benefit package) and 31 December 2016. After that, any cohort member who received an RRT modality: dialysis (either peritoneal dialysis or hemodialysis) and kidney transplant, were identified. The study also included anyone who had modality changes, mostly from peritoneal dialysis to hemodialysis, during the study period. It excluded self-paying hemodialysis patients (approximately 1,700 new UCS patients per year) and patients who had a history of temporary dialysis treatment in a period shorter than 30 days. This included patients who started dialysis then were lost to follow-up or died within 30 days; also those who were registered into the RRT programme less than 30 days before the census date (31 December 2016). Since the RRT programme was designed to cover patients with established renal failure, those who were diagnosed with acute/reversible renal failure were not included in the database.

\section{Data analysis}

Age of entry into the RRT programme was determined by the time (year) between the date of birth and the date of dialysis registration or receiving a kidney transplant. 'Cohort' was the 
individuals' years of birth. The 'period' denoted calendar years of the period studied. Individuals were grouped into 10 five-year age at registration (or at transplantation) groups (aged 20-69), and 11 five-year cohort groups (from 1942-1996). Period was used as a single year interval (from 2008-2016). The age standardised registration rates were estimated for each year of the period studied using the World Health Organization's world population in the year 2000 as the standard.

In the age-period-cohort model, a set of nested models (likelihood-ratio test) was used to compare the goodness-of-fit within a set of models. This was done by first comparing the deviance of any model that deviated from linear effects (the null model which contained only the age parameter). The deviance was then compared in this manner until the deviance of the last pair of models was obtained. The preferred model was selected by the lowest deviance. Finally, the effects of the key variables (age, period, and cohort) were estimated by using a log-linear Poisson regression. For parameterisation, drift was extracted by the weight average method. Knots (parameters) were equally spaced. Equal numbers of knots were allocated to age, period and cohort. The study used five knots in all models. Data analyses and graphical presentations were conducted by Stata version 12. Modelling was carried out using the apcfit command (Rutherford, Thompson et al. 2012). All confidence intervals are $95 \%$ confidence intervals.

\section{Results}

Overall, numbers of new patients with ESRD, aged 20-69, registered with the dialysis programme, increased over time and the total number of new registrations in the period was 56,238 (Table 1).

<Table 1 here> 
Over time, there were a larger number of cases, yet fairly constant rates in the young agegroups. Overall rates of people with dialysis registration started at 100 per million population (pmp) in 2008 (equivalent to current UK dialysis take on rates) and increased continuously over time to approximately $200 \mathrm{pmp}$ around 2014, then plateauing at that level. Despite a constant increase in dialysis take on over time, kidney transplant rates were low across all age groups, in particular for those over 60 years. The total numbers of ESRD patients who had a kidney transplant was at its highest point in 2013, at around 150 cases, then fell to just above 100 cases in 2014 to 2015 and climbed back to 150 cases again in 2016 (Table 2).

$<$ Table 2 here>

Although the registration rate in 2008 was low at the start of the programme, and the rates of all age groups were very close at first (20\%-30\% of ESRD diagnoses), by 2012, the rates had risen to a peak across most age groups and then dialysis take on gradually decreased in successive years for the age groups of 55 or more years, and particularly for the older age groups. Over the nine-year period, it is clear that the approach to dialysing older people changed, fewer older people started dialysis after the peak in 2012. Consequently, a wide gap in registration rates developed with the rates ranging from 10\%-50\% of ESRD diagnoses (Figure 1a). For kidney transplantation, the rates showed high peaks in some specific years and very young age groups (Figure 1b). These numbers need to be seen not just in the context of rates per million population, but also in terms of available facilities to deliver these interventions. Total numbers of available dialysis take-on slots have remained constant since 2015, and also the capacity for kidney transplantation. The total numbers of transplants, particularly in patients aged 40 years and above, have not changed much since 2014. In 2016 the transplant rates ranged from $0 \%-5 \%$ of ESRD diagnoses (Figure $1 \mathrm{~b}$ ).

$<$ Figure 1 here $>$ 
In the reference year 2014, the effects of age on dialysis were strongest for patients aged 2040 years. The dialysis programme could take up to 400 new patients of this age group for every 1,000 new ESRD diagnoses (Figure 2a). The dialysis programme saw many more new registrations than the transplant programme, which could treat at best only around 50 cases for every 1,000 new ESRD diagnoses in patients aged 20 (Figure 2c). From 2009, the dialysis programme showed an upward trend in registrations and the trend started to decline after 2012 (Figure 2b). For kidney transplantation, after a sharp increase between 2008 and 2010, rates increased slowly over time until 2016 (Figure 2d).

$<$ Figure 2 here>

\section{Discussion}

The RRT programme intended to provide access to essential healthcare and prevent UCS beneficiaries incurring financially catastrophic payments due to expensive treatment. It has achieved these aims by enrolling ESRD patients into the programme regardless of age or socioeconomic status and it is the NHSO who pays for service costs not patients.

The study assessed rates of registration into the RRT programme by the age-period-cohort method. The dialysis registration rate is likely to have responded to the new policy since registration trends increased after each change in reimbursement policy. Dialysis centres and professionals started to be available nationwide from 2009 onwards (NHSO 2017). With availability of services, previously unrecognised cases with ESRD were identified and registered. Hence, registration rates began to increase sharply in the 24 months following the introduction of the programme in 2008. An increase was also evident when the NHSO started to reimburse treatment for UCS patients who had previously refused the PD-first policy, and 
allowed them to register for free hemodialysis. This additional benefit is likely to have boosted the dialysis registration rate after 2014 .

It was evident that not all patients started RRT once they were newly diagnosed with ESRD. More than half of all ESRD patients were not registered into the dialysis programme. In addition, after dialysis registrations reached a peak in 2012, the RRT programme was able to enroll a higher percentage of young patients needing RRT; however the registration rates tended to decrease with age, despite the high and growing proportion of older patients with ESRD diagnoses.

There are two likely explanations of these findings. Firstly, the guideline of taking up patients into the RRT programme developed by the Thai Nephrology Society recommended that patients with ESRD diagnoses without signs or symptoms of kidney failure should be asked to register into the RRT programme when their eGFR levels dropped to $6 \mathrm{ml} /$ minute per 1.73 $\mathrm{m}^{2}$. The principle of waiting until the eGFR was low was based on the IDEAL trial (Cooper et al. 2010).

Secondly, for the low registration rates in elderly patients, the finding corresponds to that of some prior studies (Morton et al. 2010; Rayner et al. 2014; Tonkin-Crine et al. 2015; Thammatacharee 2016) that the elderly with ESRD, who have multiple illnesses and lack family support, are likely to be unable to use or may refuse to start dialysis. This is less likely to be a supply side issue, since in the UCS, the guideline of RRT registration does not indicate an age limit and the payment to the RRT programme is based on the number of registrations. So the more patients are registered, the greater the amount of reimbursement to dialysis centres.

When comparing RRT with other health care interventions, RRT modalities are usually ranked as providing inferior value for money expressed in terms of incremental cost- 
effectiveness ratios (ICER) (Chaikledklew 2014). Thailand took the decision to include RRT in the UCS benefit package due to concern about inequalities with the health benefit packages of other social insurance schemes, and evidence of the impoverishing effects of payment for treatment for affected people and their families. Results from other literature have indicated that HD is the least cost-effective treatment option, while continuous ambulatory peritoneal dialysis and kidney transplant represent more cost-effective approaches (Sennfalt et al. 2002; Kontodimopoulos and Niakas 2008; Haller et al. 2011). While renal dialysis is less costeffective in comparison to transplantation (Muirhead 1996) but it is essential to prolong a patient's life and since organ donors tend to be in short supply, transplantation is not available to every patient.

Thailand has introduced many strategies to promote organ donation, for example patients are asked on admission whether they wish to donate their organs, an online organ registration system has been set up, and a number of organ donation campaigns launched. These strategies have encouraged growth in the number of donations, in particular from deceased donors (Thai Transplantation Society 2017). Nonetheless the kidney transplant rate in Thailand was approximately 8 pmp (Chuasuwan and Praditpornsilpa 2015) which was low in comparison to other countries worldwide (GODT 2017). This study found that the kidney transplant rate of UCS patients was 3 pmp. At this rate, it accounted for just one-third of all kidney transplants in Thailand, despite the fact that the UCS is the largest public health scheme and covers $76 \%$ of all Thais.

This study found that the incidence of RRT registrations among UCS patients was very high (200 pmp). Assuming that this number accounted for 50\% of all ESRD patients, the incidence of ESRD in Thailand may be one of the highest in the world, comparable to countries with the highest ESRD incidence like Taiwan (476 pmp), the Jalisco region of Mexico (411 pmp), and the United States (376 pmp) (United States Renal Data System 2017). This highlights 
that chronic kidney disease is very important for Thailand, and that prevention of people requiring dialysis is needed. Especially for diabetes there is good evidence that the need to dialysis can be prevented by blood pressure and diabetes treatment.

A systematic review estimated that around the world in 2010, there were 9.7 million patients needing renal replacement therapy (RRT) but only 2.6 million patients were able to use it. The proportion of those in need but not receiving RRT ranged from just 5\% in North America to $91 \%$ in Africa (Liyanage et al. 2015). Variations among different regions were found to be more associated with supply-side factors (such as macroeconomic issues, health systems, and renal service provision) rather than health status or demographics of the general population (Caskey et al. 2011).

Evidence of inequalities due to the insurance system and RRT provision also exists in other lower-middle income countries. For example in Mexico, chronic kidney disease was a serious health problem but only Social Security System beneficiaries (48\% of the overall population) had access to RRT benefits. The public insurance programme that covered the rest of population did not include RRT services (Pecoits-Filho et al. 2009; Garcia-Garcia and Chavez-Iñiguez 2018). In Malaysia, the government is the main source of funding for dialysis (63\%). These funds are channeled as subsidies to non-profit HD centres and as payment for dialysis treatment for pensioners, civil servants and their dependents in private HD centres. Patients without these subsidies pay themselves or receive charitable support (Ahmad et al., 2015). Thailand introduced the current service on the grounds that it would save lives and prevent indebtedness and health impoverishment among UCS members and their families.

In Thailand, chronic kidney disease was not ranked as a topmost health problem in terms of deaths and disability. It was reported to be an underlying condition in patients with hypertension, diabetes, high uric acid, and using traditional medicines (Ingsathit et al. 2009). 
It is not surprising, after all chronic kidney disease is frequently asymptomatic until patients reach ESRD, and in the absence of diagnostic services may be misdiagnosed as another condition. Apart from hypertension and diabetes, chronic glomerulonephritis was one of the main primary diagnoses among those who had ESRD and later underwent kidney transplant (Thai Transplantation Society 2017). The government has been launching many health promotion and prevention programmes to control non-communicable diseases but it is too early to look for positive results in relation to ESRD from these programmes, as ESRD develops over decades in frequently asymptomatic individuals.

This study was a facility-based study which enhanced validity by capturing most individuals of interest and results were representative of the studied population. In addition, the ageperiod-cohort analysis is regarded as a powerful tool in modelling and analysing routinely collected information from administrative records or disease registries (Carstensen 2007). This study has some limitations. The age-period-cohort analysis does not establish a causeeffect relationship to prove whether the RRT programme really caused an effect on the outcome of interest (registration). In addition, the secondary data used in this analysis were mainly intended for administrative purposes, and thus had limitations. For example, data for many fields which were not used for budget claims, such as laboratory results, were not routinely recorded.

To date, there is still a lack of reliable epidemiological information on the various stages of chronic kidney disease in the general population. This is because it is a silent disease: a patient may be living asymptomatically until the condition reaches an advanced stage. Patients who receive RRT can be used to identify the number of patients with last-stage chronic kidney disease, however numbers of patients in this sense reflect only the portion of patients who receive treatment and cannot be used to represent the entire 
affected population, or even the number of patients with ESRD (Eustace and Coresh 2005).

\section{Conclusions}

Thailand is unusual as a middle income country in having opened access to RRT for its whole population. It did this by including RRT in the benefit package of the UCS and expanding treatment resources. This paper provides evidence that rates of registration increased, especially for younger groups whereas older people may have been less willing to enter treatment and may benefit from conservative management programmes. Transplantation rates remain too low, and it is hoped that kidney live-donation programmes can be established. Policy makers should find strategies to reduce the increasing number of patients with kidney disease who later develop ESRD.

\section{Acknowledgements}

We thank the NHSO for providing data and Martin McKee, Supon Limwattananon, and Chulaporn Limwattananon for their suggestions about the methodology. This work was supported by the International Health Policy Program.

Conflict of interest statement. D.N. is involved in GSK funded research of CKD in Sub Saharan Africa.

\section{References}

Ahmad G, Leong GB, Ngo LY, et al. 2016. Dialysis in Malaysia. In: Wong HS, Goh BL (eds). 24 ${ }^{\text {th }}$ Report of the Malaysian Dialysis and Transplant Registry: National Renal Registry 2016.

Caskey FJ et al. 2011. Global variation in renal replacement therapy for end-stage renal disease. Nephrol Dial Transplant 26(8):2604-2610.

Carstensen B. 2007. Age-period-cohort models for the Lexis diagram. Statist Med 26: 30183045.

Chaikledklew U. 2014. Assessing cost and cost effectiveness, Paper presented at Asia-Pacific Economic Cooperation Workshop, Manila, 10-11 April 2014. 
Chuasuwan A, Praditpornsilpa K (eds). 2015. Thailand Renal Replacement Therapy. The Nephrology Society of Thailand.

Chungsaman P, Kasemsap P. 2017. PD First Policy: Thailand's Response to the Challenge of Meeting the Needs of Patients With End-Stage Renal Disease. Semin Nephrol. 37(3): 287295.

Cooper B et al. 2010. A Randomized, Controlled Trial of Early versus Late Initiation of Dialysis. N Engl J Med 363: 609-619.

Coresh J, Jafar T. 2015. Disparities in worldwide treatment of kidney failure. The Lancet 385(9981): 1926-1928.

Garcia-Garcia G, Chavez-Iñiguez JS. 2018. The Tragedy of HavingESRD in Mexico. Kidney Int Rep 3:1027-1029.

Grassmann A et al. 2005. ESRD patients in 2004: global overview of patient numbers, treatment modalities and associated trends. Nephrol Dial Transplant 20(12): 2587-2593.

Haller M et al. 2011. Cost-effectiveness analysis of renal replacement therapy in Austria. Nephrol Dial Transplant 26(9): 2988-2995.

Ingsathit A et al. 2009. Prevalence and risk factors of chronic kidney disease in the Thai adult population: Thai SEEK study. Nephrol Dial Transplant 25(5): 1567-1575.

Jah V. 2013. Current status of end-stage renal disease care in India and Pakistan. Kidney Int Supp/ 3: 157-160.

Kontodimopoulos N, Niakas D. 2008. An estimate of lifelong costs and QALYs in renal replacement therapy based on patients' life expectancy. Health Policy 86(1): 85-96.

Liyanage T et al. 2015. Worldwide access to treatment for end-stage kidney disease: a systematic review. The Lancet_385(9981): 1975-1982.

Morton R et al. 2010. The views of patients and carers in treatment decision making for chronic kidney disease: systematic review and thematic synthesis of qualitative studies. $B M J$ 340: $c 112$.

Muirhead N. 1996. A study of the quality of life and cost-utility of renal transplantation. Kidney Int 50: 235-42.

NHSO. 2017. Annual Report Bangkok, Thailand, National Health Security Office.

Odubanjo $\mathrm{M}$ et al. 2011. The epidemiology of end-stage renal disease in Nigeria: the way forward. Int J Urol_43(3): 785-792.

Pecoits-Filho R et al. 2009. Policies and health care financing issues for dialysis in Latin America: extracts from the roundtable discussion on the economics of dialysis and chronic kidney disease. Peri Dial Int 29 (Supplement 2): S222-S226.

Rayner $\mathrm{H}$ et al. 2014. Does community-wide chronic kidney disease management improve patient outcomes? Nephrol Dial Transplant 29(3): 644-649.

Rutherford M et al. 2012. Projecting Cancer Incidence using Age-period-cohort Models Incorporating Restricted Cubic Splines. Int J Biostat Retrieved 14 May. 2018 from doi:10.1515/1557-4679.1411. 
Sakhuja V, Kohli H. 2006. End-stage renal disease in India and Pakistan: incidence, causes, and management', . Ethn Dis 16(2): S2.

Sennfalt $\mathrm{K}$ et al. 2002. Comparison of hemodialysis and peritoneal dialysis--a cost-utility analysis. Perit Dial Int 22(1): 39-47.

Tangcharoensathien V et al. 2005. Universal Access to Renal Replacement Therapy in Thailand : a policy analysis. Nonthaburi., Health System Research Office.

Thai Transplantation Society. 2017. Annual Report 2016. Bangkok.

Thammatacharee N. 2016. Can a disease management approach facilitate the inclusion of high-cost conditions in a benefit package?: the case of renal replacement therapy in Thailand. Doctoral dissertation, University of London. London.

Tonkin-Crine $S$ et al. 2015. Understanding by older patients of dialysis and conservative management for chronic kidney failure. Am J Kidney Dis 65(3): 443-450.

United States Renal Data System. 2017. Chapter 11: International Comparisons. Annual Data Report: Epidemiology of Kidney Disease in the United States. MD, National Institutes of Health, National Institute of Diabetes and Digestive and Kidney Diseases, Bethesda.

White $S$ et al. 2008. How can we achieve global equity in provision of renal replacement therapy? Bull World Health Organ 86(3): 229-237.

Leong GB et al. 2015. Dialysis in Malaysia In: Wong HS, Goh BL (eds). 23 ${ }^{\text {rd }}$ Report of the Malaysian Dialysis and Transplant Registry: National Renal Registry 2015.

Table 1 Numbers of dialysis registrations ( $n$ ) and age-standardised rates of dialysis registration for patients aged 20-69 years, 2008-2016

\begin{tabular}{|c|c|c|c|c|c|c|c|c|c|}
\hline \multirow[b]{2}{*}{ Year } & \multicolumn{2}{|c|}{$20-39$ yrs } & \multicolumn{2}{|c|}{$40-59$ yrs } & \multicolumn{2}{|c|}{$60-69$ yrs } & \multicolumn{3}{|c|}{ Overall } \\
\hline & $\mathrm{n}$ & Rate* & $\mathrm{n}$ & Rate* & $\mathrm{n}$ & Rate* & $\mathrm{n}$ & Crude rate* & $\begin{array}{l}\text { Adjusted } \\
\text { rate* }\end{array}$ \\
\hline 2008 & 604 & 49 & 2139 & 170 & 417 & 131 & 3160 & 113 & 103 \\
\hline 2009 & 467 & 38 & 1718 & 133 & 407 & 122 & 2592 & 91 & 83 \\
\hline 2010 & 601 & 49 & 2503 & 191 & 812 & 232 & 3916 & 136 & 123 \\
\hline 2011 & 735 & 62 & 3036 & 227 & 1238 & 330 & 5009 & 173 & 154 \\
\hline 2012 & 824 & 67 & 3496 & 258 & 1732 & 454 & 6052 & 204 & 182 \\
\hline 2013 & 1039 & 86 & 4371 & 321 & 2556 & 638 & 7966 & 268 & 235 \\
\hline 2014 & 1029 & 51 & 4714 & 242 & 3087 & 595 & 8830 & 198 & 183 \\
\hline 2015 & 1063 & 54 & 4867 & 248 & 3458 & 637 & 9388 & 209 & 191 \\
\hline 2016 & 1069 & 54 & 4771 & 240 & 3485 & 611 & 9325 & 206 & 186 \\
\hline
\end{tabular}

*rate per million UCS population years within each age group 
Table 2 Numbers of new kidney transplants ( $n$ ) and age-standardised rates of kidney transplants for patients aged 20-69 years, 2008-2016

\begin{tabular}{|c|c|c|c|c|c|c|c|c|c|}
\hline \multirow[b]{2}{*}{ Year } & \multicolumn{2}{|c|}{ 20-39 yrs } & \multicolumn{2}{|c|}{$40-59$ yrs } & \multicolumn{2}{|c|}{$60-69$ yrs } & \multicolumn{3}{|c|}{ Overall } \\
\hline & $\mathrm{n}$ & Rate* & $\mathrm{n}$ & Rate* & $n$ & Rate* & $n$ & Crude rate* & Adjusted rate* \\
\hline 2008 & 7 & 1 & 3 & 0 & 0 & 0 & 10 & 0 & 0 \\
\hline 2009 & 17 & 1 & 10 & 1 & 1 & 0 & 28 & 1 & 1 \\
\hline 2010 & 27 & 2 & 17 & 2 & 1 & 0 & 55 & 2 & 2 \\
\hline 2011 & 40 & 3 & 24 & 2 & 2 & 1 & 66 & 2 & 2 \\
\hline 2012 & 29 & 2 & 25 & 2 & 4 & 1 & 58 & 2 & 2 \\
\hline 2013 & 61 & 5 & 76 & 6 & 9 & 2 & 146 & 5 & 5 \\
\hline 2014 & 40 & 2 & 69 & 4 & 10 & 2 & 119 & 3 & 3 \\
\hline 2015 & 54 & 3 & 62 & 3 & 6 & 1 & 122 & 3 & 3 \\
\hline 2016 & 65 & 3 & 78 & 4 & 8 & 1 & 151 & 3 & 3 \\
\hline
\end{tabular}

*rate per million UCS population years within each age group

Figure 1 Figure 1 Dialysis registration (a) and kidney transplant (b) rates per 1000 ESRD diagnoses* by year and age at diagnosis, 2008-16.
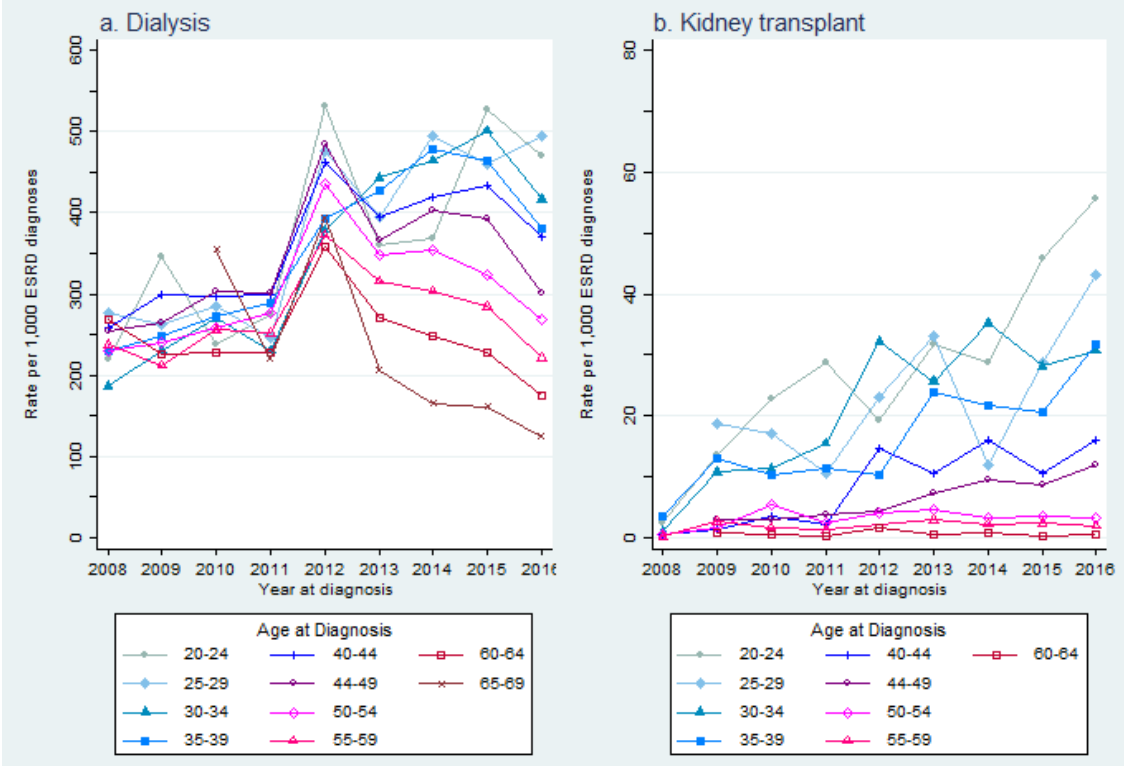
Figure 2 Effects of age ( $a$ and $c$ ) and period ( $b$ and $d$ ) on dialysis registrations and transplant (rates per 1000 ESRD diagnoses).
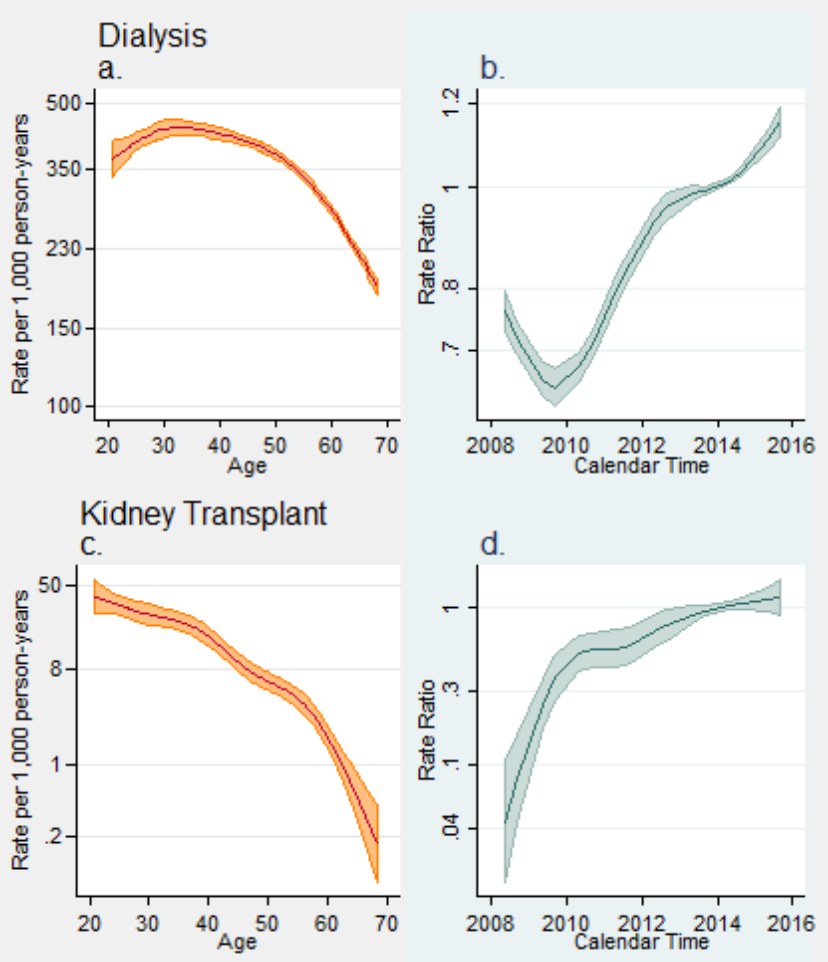